\title{
Research on the Tail Dependence of Agriculture Listed Companies
}

\author{
Pei-song $\mathrm{Mu}$ \\ School of Economic Administration, Sichuan Agricultural University \\ Ya'an, 625014, China
}

Tel: 86-135-5894-0649Ｅ-mail: mupeisong@139.com

Xun-gang Zheng (Corresponding author)

School of Economic Administration, Sichuan Agricultural University

Ya'an, 625014, China

Tel: 86-130-8630-5086 E-mail: zxg9@163.com

\begin{abstract}
Based on the Conditional Probability Model of Gumbel-H Copula and Clayton Copula distribution function to measure the tail dependence of financial asset, and the interior relationship between these two types of Copula distribution functions and Kendall $\tau$, this article calculates the tail dependence of agricultural listed companies in Shanghai and Shenzhen by the non-parametric estimation method. The results shows that the tail dependence is existed between Shanghai and Shenzhen listed companies in agriculture, and the four kinds of listed companies in Shanghai which are farming, forestry, animal husbandry and fishery respectively have the tail dependence with the same four kinds of companies in Shenzhen. In addition, all tail dependence is asymmetry.
\end{abstract}

Keywords: Copula functions, Agricultural listed companies, Tail dependence, Non-parametric estimation

\section{Introduction}

With the development of times, the mutual dependence and influence among the financial market growing with each passing day, gregariousness and continuity are more and more obvious in stock market. Cluster refers to a greater probability of large fluctuations will be presented after a large volatility, and the greater probability of small fluctuations will be also presented after a small volatility. The emergence of clustering often affects the stability of the stock market, giving some investors huge profits, while giving some other investors a huge loss. Due to the uncertainty of its occurrence on the "clustering", it is difficult to study on it. In 1959, Sklar puts forward that a joint distribution can be decomposed into its k-edge distribution and a Copula function, then the Copula functions describe the correlation structure between the variables. Subsequently, the theory is applied to study the volatility of financial data by many scholars, and "tail dependence" of the model between random variables is presented. As a result, it provides a new thoroughfare for the study of "cluster" in stock market.

The main purpose of the study on tail correlation is to determine how the random variable changes while other random variables change. The size of the tail correlation coefficient reflects the size of probability of the possibility of simultaneous extremum among observed variables. For example, the tail correlation between the Shanghai and Shenzhen stock market could reflect the simultaneously possibility of big fluctuations of two stock markets; If the research is the correlation between individual stocks, the tail correlation reflects the twists and turns of a stock whether accompanied by another stock spike. For investors, if a variety of stocks that he held fell slightly at the same time, it may not cause much impact, but if these stocks steeply fall at the same time, it will give investors a huge economic loss. The tail correlation can be seen as the real concerns to investors and the focal point in relevance study of risk analysis.

At present, the study on tail copula function focused on two aspects that measuring the nature of the tail correlation among various kinds of copula function and its application in the stock market. LI Yue, CHENG $\mathrm{Xi}$-jun (2005) analyzed the tail correlation of connectivity function (copula) and revealed the relevance of the Shanghai Index and the Heng Sheng Index, holding that quantified correlation can predict the stock market change; Wang Lu, Wang Qin, Pang Hao(2007)introduced the left tail (right tail) correlation coefficient, discussed their Copula Measures and related properties, analyzed the Shanghai and Shenzhen stock index yield trends in 
the tail correlation through the computer simulation, and then found out that the Shanghai and Shenzhen stock index return of tail dependence is enhanced and asymmetric; ZHONG Jun, SHI Dao Ji (2008) studied the tail correlation function of the Shanghai and Shenzhen stock markets'returns, the result showed that there was a strong tail dependence in the logarithmic returns; WANG Xiao-fang, XIE Jin (2009) had a comparative study on the tail correlation of return series between Shanghai Composite index and Singapore's Straits Times index by static and dynamic Copula functions, which found that the tail correlation of return series is asymmetry, and lower tail correlation is more notable than upper tail correlation; CHEN Chong-shuang, MA Li-qiong, WU Hong-tao, et al (2008) studied on the tail correlation of the parameters family of Archimedean Copula, proposing the conclusion that the tail correlation coefficient of the parameters for high-dimensional and two-dimensional Archimedean Copula family are the same; HU LI-xia (2008) studied a few properties of the lower tail dependence Copula.

In the studies of copula function parameter estimation, there are mainly the parameter estimation of determining the marginal distribution and non-parametric estimation of uncertainty in the edge of the distribution. Parameter estimation has a more stringent requirement on the marginal distribution. If the edge of the function is correct, then the results are very reliable, but if the edge of the function is error, the next analysis will be meaningless. The non-parameter estimation method avoids this shortcoming, so non-parametric estimation of the estimation method has become the mainstream. LI Yue, CHENG Xi-Chun (2005), ZHONG Jun, SHI Ji-Tao (2008), REN Xian-ling, ZHANG Shi-ying(2008) et al. all achieved the parameter estimates by applying non-parametric estimation method.

Agriculture is not only the basis for the national economy and its development, but also the basis for social stability. Agricultural listed company as a leading agricultural industrialization is an important carrier to solve the contradictions of "small farmers" and "big market", and achieve industrial management of agriculture. The development of agricultural listed companies plays an important role in the development of the entire agricultural economy. Currently, the research of agricultural listed companies has focused on the following aspects:

(1) Research on business performance: LIN Le-fen(2004)'s study showed that agricultural listed company's own performance is general low, existing relatively large public space, but science and technology enterprises and deep processing enterprises performed better relatively. LIU Wei, YANG Yin-sheng (2006)'s study suggested that agricultural listed companies are relatively weak in the solvency and asset management capability; cash capacity, the ability grows relatively strong, so the potential for development of agricultural listed companies is still relatively large.

(2) Research on "Back agricultural investment": LIU Zhu-lin, HU Yun-xia (2008)'s study showed that Defects such as inefficient agriculture, a long period for input, low profit margins lead to"back agricultural investments". However, the main reason to lead to "farmers back" is the attitudes and practices of enterprises and government of agricultural listed companies and their own governance structure; GAO Jia-kuan, et al (2008) comparatively studied on the "farmers back" and "non-back farm" enterprises, saying that the overall operating performance of agricultural listed company of "farmers back" category is lower than the "non-back farm" category, while the business risk is higher than "non-back farmers."

(3) Research on Equity Financing: HUANG Yao, CAO Dong-xin (2004)'s study shows that with the process of agricultural industrialization and increasing in comparative advantage in agriculture, agriculture funded lacking effective demand has gradually become less effective supply. With the constraints of the indirect financing channels tending to harden, to raise funds through securities markets has become an effective channel for agricultural companies. CHEN Dong-ping, ZHANG Jing-ming (2006) studied the dividend policy of agricultural listed companies, which showed that the per-share stock dividend, investor's preference, refinancing ability and the company's growth capacity has a significant influence to the cash dividends; investor preferences, the demand for currency and capital, refinancing capacity, equity concentration and the ability to grow have a significant impact on stock dividends; To Chinese agricultural listed companies, it is more steady on cash dividend distribution than stock dividends; SUN Bei-bei, ZHONG Jian -xin (2007)'s research shows that agricultural listed companies in China financing order is the first external finance, and then endogenous financing, mainly relying on external financial. Agricultural listed Companies in China, whether endogenous or exogenous financing, all need to raise their financing ability. A weak financing capacity is vulnerable to hit by financial bottleneck, leading difficult to expansion firm size and form scale effect, and further affecting the stability and sustainable development of listed companies in agriculture.

To sum up, copula functions do have their unique advantages on the tail of the variable correlation studies. 
However, there is still a huge space with the copula function to measure the tail of the agricultural correlation between listed companies. In this article, the research on measuring the stock market tail copula function is applied in agriculture listed companies, applying of non-parametric estimation methods, efforts to explore the tail dependence of Shanghai and Shenzhen-listed A-share listed companies in agriculture, and the tail correlation of farming, forestry, animal husbandry, fishery type in Shanghai and Shenzhen agricultural listed companies.

\section{Copula functions and non-parametric estimation}

\subsection{The measure of Copula tail correlation}

The tail correlation is measured when a random variable increases or reduces significantly, the probability of the random variables also increased or reduced significantly (A. Juri, 2002). The tail correlation is divided into the upper tail dependence and lower tail dependence. Making random variable vector as sequence of random variables, function $\mathrm{F}$ is the marginal density function of $\mathrm{x}$, that is $U=F(x)$, function $\mathrm{G}$ is the marginal density function of y,that is $V=G(y), \alpha$ is a specific value, its tail coefficient is defined as follows:

2.1.1 The upper tail correlation coefficient

$$
\lambda_{U}=\lim _{\alpha \rightarrow 1^{-}} P(G(y)>\alpha \mid F(x)>\alpha)
$$

If $\lambda_{U}>0,(x, y)$ is upper tail correlation; $\lambda_{U}=0,(x, y)$ is upper tail independence.

2.1.2 The lower tail correlation coefficient

$$
\lambda_{L}=\lim _{\alpha \rightarrow 0^{+}} P(G(y) \leq \alpha \mid F(x) \leq \alpha)
$$

If $\lambda_{L}>0,(x, y)$ is upper tail correlation; If $\lambda_{L}=0,(x, y)$ is upper tail independence.

If $\lambda_{U}, \lambda_{L}>0,(x, y)$ is tail correlation.

\subsection{Copula Functions}

\subsubsection{Gumbel-H copula Functions}

The expression of distribution function is as follows:

$$
C^{G u}(u, v, \theta)=\exp \left[-\left((-\ln (u))^{\theta}+(-\ln (v))^{\theta}\right)^{1 / \theta}\right], \theta \geq 1
$$

The function is sensitive to the changes of the variable in the upper tail distribution so that the changes on upper tail correlation can be captured quickly, which can be used to describe the correlation among the financial markets with the characteristics of the upper tail dependence. Parameter $\theta$ describes the degree of correlation, when $\theta=1$, variables is independence; when $\theta \rightarrow \infty$, variables are fully relevant. The extremum "copula" is Gumbel-H copula, which is able to capture the risk information in a bear or bull market period. With upper tail correlation coefficient $\lambda_{U}, \theta$ is the analytical function of $\tau$, and $\tau$ is the related coefficient of Kendall $\tau$ :

$$
\theta=1 /(1-\tau)
$$

By (1) we can get $\lambda_{U}(\alpha)$ of Gumbel-H copula function in different level of $\alpha$, which is defined as the left limit:

$$
\begin{aligned}
\lambda_{U} & =\lim _{\alpha \rightarrow 1^{-}} P\left(G^{-1}(y)>\alpha \mid F^{-1}(x)>\alpha\right) \\
= & \lim _{\alpha \rightarrow 1^{-}}\left(1-2 \alpha+C^{G u}(\alpha, \alpha)\right) /(1-\alpha)
\end{aligned}
$$

\subsubsection{Clayton copula Functions}

The expression of distribution function is as follows: 


$$
C^{C l}(u, v, \theta)=\left(u^{-\theta}+v^{-\theta}-1\right)^{-1 / \theta}, \theta>0
$$

The function is sensitive to the changes of the variable in the lower tail distribution so that the changes on lower tail correlation can be captured quickly, which can be used to describe the correlation among the financial markets with the characteristics of the lower tail dependence. When $\theta \rightarrow 0$ and $\theta \rightarrow \infty$, it represents respectively variables is independence and fully consistent with the relevant variables. With a correlation coefficient of lower tail $\lambda_{L}, \theta$ is the analytical function of $\tau$ :

$$
\theta=2 \tau /(1-\tau)
$$

By (2) we can get $\lambda_{U}(\alpha)$ of Gumbel-H copula function in different level of $\alpha$, which is defined as the right limit:

$$
\begin{gathered}
\lambda_{L}=\lim _{\alpha \rightarrow 0^{+}} P\left(G^{-1}(y) \leq \alpha \mid F^{-1}(x) \leq \alpha\right) \\
=\lim _{\alpha \rightarrow 0^{+}} C^{C l}(\alpha, \alpha) / \alpha
\end{gathered}
$$

\subsubsection{Non-parametric Estimation of $\theta$}

Calculating Kendall $\tau$ correlation coefficient after estimating the value of parameter $\theta$, it is defined as follows:

$$
\tau=P\left[\left(x_{i}-x_{j}\right)\left(y_{i}-y_{j}\right)>0\right]-P\left[\left(x_{i}-x_{j}\right)\left(y_{i}-y_{j}\right)<0\right]
$$

$\left(x_{i}-x_{j}\right)$ and $\left(y_{i}-y_{j}\right)$ is independent and identically distributed variables for the sample of $\mathrm{n}$. Fitting Kendall $\tau$ correlation coefficient is as follows:

$$
\begin{gathered}
\hat{\tau}(x, y)=\frac{2(n-2) !}{n !} \sum_{1 \leq i<j \leq n} \operatorname{sign}\left[\left(x_{i}-x_{j}\right)\left(y_{i}-y_{j}\right)\right] \\
\operatorname{sign}(x)=\left\{\begin{array}{l}
1 x>0 \\
0 x=0 \\
-1 x<0
\end{array}\right.
\end{gathered}
$$

Then, $\hat{\theta}$ can be calculated by estimating $\hat{\tau}$.

\subsubsection{Copula Function Tests}

In 2000, V. Durrleman has defined distribution functions as follows:

$$
k_{C}(t)=P(C(u, v) \leq t)=t-\varphi(t) / \varphi^{\prime}(t)
$$

$\varphi(t)$ is the generating function of copula function, in which $k_{C}(t)$ subjects to the standard uniform distribution and can be calculated by using $\hat{\theta}$.

For the Gumbel-H copula:

$$
k_{C}^{G u}(t)=t-\ln (t) t / \theta
$$

For the Clayton copula:

$$
k_{C}^{C l}(t)=t+\left(t^{-\theta}-1\right) /\left(\theta t^{-\theta-1}\right)
$$

The steps that select the appropriate copula are as follows:

(1) Calculate edge function $F(x)$ of random vector $(x, y)$ and empirical distribution function $G(y)$;

(2) Calculate $t^{G u}=C^{G u}(F(x), G(y))$ and $t^{C l}=C^{C l}(F(x), G(y))$; 
(3) $t$ that calculated from (2) is substituted into (13) and (14);

(4) $k_{C} \sim U(0,1)$, tested by $K-S$

\section{Data description and estimation results of model}

In 2007 and 2008, there are 35 listed companies related to farming, forestry, animal husbandry, and fisheries in Shanghai and Shenzhen. Due to incomplete data of ten listed companies, this paper selects 25 companies as the object of study, showing from the table 1 .

The data is needed to process before the analysis as follows:

First of all, to January 4, 2007 as the base year, it calculates various stock's closing price index by Paramount Index Method:

$$
I=\sum_{i=1}^{n} p_{1 i} q_{1 i} / \sum_{i=1}^{n} p_{0 i} q_{1 i}
$$

${ }_{1 i}$ is the closing price of reporting period; $p_{0 i}$ is the closing price of base period; $q_{1 i}$ is stock turnover of reporting period.

Secondly, because logarithmic rate of return can be applied in any case, and the calculation is simple, more suitable for the establishment of financial assets of behavioral model, it is used in this paper. The formula is $R=100\left(\ln \left(I_{1}\right)-\ln \left(I_{0}\right)\right)$, in which $I_{1}$ is today's closing index, $I_{0}$ is yesterday's closing index.

Finally, the results calculated by Matlab 7.0 are as table 2 to table 4 .

\section{Interpretation of result}

\subsection{K-S tests and analysis}

$\mathrm{K}-\mathrm{S}$ test is a common test that measures the reasonable of copula function and strengths or weaknesses of a variety of copula function. It has two parameters that one is the K-S test value, and the other is the corresponding probability values. If the larger K-S test value is, the smaller the corresponding probability is. Therefore, given the significant level, Copula Function whose probability value is greater than the specified significance level is reasonable. At a reasonable premise, the smaller K-S test value of the copula function is, the better they can respond the nature of research object more realistically. From Table 2 and Table 3 , at 0.01 significant level, the probability value of Gumbel-H copula and Clayton copula is greater than 0.01 that sets in this paper, which shows that it is reasonable to research the tail correlation of agricultural listed companies in Shanghai and Shenzhen by Copula Function.

\subsection{Analysis of the tail correlation of Agricultural Listed Companies}

\subsubsection{There is tail correlation between agricultural listed companies in Shanghai and Shenzhen as a whole}

It's not difficult to conclude from the table 2, 3 and 4 that Gumbel-H parameter value 1.3849 is greater than 1, then Clayton parameter value 0.7699 is greater than 0 , which shows that the upper and lower tail correlation is existed in agricultural listed companies of Shanghai and Shenzhen, that is the two tail-related. And from Table 4 it can be further quantified their correlation of structure: based on the definition of the upper tail correlation it can be seen that at the level of $0.05,0.025,0.005$, the logarithm of rate of return in agricultural listed companies of Shenzhen is greater than $0.95,0.975,0.995$; under the conditions of corresponding quantile, the logarithm of rate of return in agricultural listed companies of Shanghai is also greater than $0.95,0.975,0.995$; the probability of the corresponding quantile of $0.3774,0.3639,0.3531$ is greater than the independent probability of $0.05,0.025,0.005$, which shows that the upper tail correlation is existed and its correlation coefficient of Shanghai and Shenzhen is 0.3504.That is to say, on the condition of the increased of logarithmic return rate in Shenzhen-listed agricultural companies, increasing in the probability of logarithmic return rate in Shanghai-listed agricultural companies is 0.3504.In a similar way, based on the definition of the lower tail correlation it can be seen that the lower tail correlation is existed in Shanghai and Shenzhen-listed agricultural companies and its correlation coefficient of 0.4064 , which means that on the condition of the decrease of logarithmic return rate in Shenzhen-listed agricultural companies, decreasing in the probability of logarithmic return rate in Shanghai-listed agricultural companies is 0.4064 .

4.2.2 There are different degrees of tail dependence in four types of agriculture-related listed companies in Shanghai and Shenzhen 
The above analysis is the overall tail dependence of Shanghai and Shenzhen listed companies in agriculture, and the next analysis is the tail dependence of four types of agriculture-related listed companies in Shanghai and Shenzhen from the partial. From the coefficient of Gumbel-H and copula Clayton copula in table 2 and 3, it can be seen that the upper and lower tail correlation is existed in four types of agriculture-related listed companies, and the probability values are greater than the independent probability of $0.05,0.025,0.005$. From the upper and lower tail related coefficient in table 4 it can be concluded that the upper and lower tail related coefficient of 0.5317 and 0.6500 respectively in farming listed companies is the largest in all types of agriculture-related listed companies in Shanghai and Shenzhen, which shows that the probability of logarithmic return rate rise and fall simultaneously in farming listed companies of Shanghai and Shenzhen is 0.5317 and 0.6500 respectively. Secondly, the upper and lower tail related coefficient in forestry listed companies in Shanghai and Shenzhen is 0.2360 and 0.2086 respectively, which means that the probability of logarithmic return rate rise and fall simultaneously in forestry listed companies in Shanghai and Shenzhen is 0.2360 and 0.2086 respectively. At last, the upper and lower tail related coefficient in animal husbandry listed companies in Shanghai and Shenzhen is 0.1567 and 0.0745 respectively, which also indicates that the probability of logarithmic return rate rise and fall simultaneously in animal husbandry listed company in Shanghai and Shenzhen is 0.1567 and 0.0745 respectively.

4.2.3 Tail correlation is asymmetry in agriculture listed companies and in four types of agriculture-related listed companies in Shanghai and Shenzhen

From table 4 it can be seen that the upper tail coefficient is less than the lower tail coefficient in agricultural listed companies in Shanghai and Shenzhen, which shows that compared with the upward trend of logarithmic rate of return, the declining trend is more relevant. That is to say, the probability of decrease simultaneously is greater than the probability of simultaneously increasing in agricultural listed companies in Shanghai and Shenzhen. In a similar way, among four types of agriculture-related listed companies in Shanghai and Shenzhen, the situation that upper tail coefficient is less than lower tail coefficient is existed in farming listed companies, which shows that comparing with the upward trend of logarithmic rate of return simultaneously, the declining trend simultaneously is more relevant in farming listed companies in Shanghai and Shenzhen, whereas the upper tail coefficient of the remaining three types is greater than the lower tail coefficient. From the probability values in various companies it can be seen that the upper and lower tail related coefficients are not equal between them, so the tail correlation is asymmetric in agricultural listed companies and in four types of agriculture-related listed companies in Shanghai and Shenzhen.

In a word, through analyzing the tail dependence of agricultural listed companies and four types of agriculture-related listed companies in Shanghai and Shenzhen, it is beneficial for risk managers to grasp various tail correlation of the investment in agricultural assets reasonably, thus achieve the aim to reduce the investment risks. However, fluctuations in yield will lead to the tail of the relevance of changes. At present, stock market is immature in China, and a variety of institution is needed to be improved further. The change of the return rate of agricultural listed companies is limited not only by constraints from internal governance structure, but also by the policies and external factors such as natural disasters. Therefore, the listed companies should strengthen and improve the system of internal governance, such as company's personnel structure, technology management and technology innovation. At the same time, government departments also need to establish the free competition in the financial market system, rational foreign investment environment and the financial regulatory system on agricultural listed companies, with a view to provide a good external financing environment for listed companies of agriculture.

\section{References}

CHEN Chong-shuang, MA Li-qiong, WU Hong-tao, et al. (2008). Tail dependence of Archimedean Copula with parameter. Journal of Southwest University for Nationalities (Natural Science Edition), 34(5):921-925.

CHEN Dong-ping, ZHANG Jing-ming. (2006). Influence factors analysis on dividend policy of listed agricultural companies. Journal of Nanjing Agricultural University (Social Sciences Edition), 6(2):25-30.

GAO Jia-kuan, LIN Hong, GUO Ni-ya. (2008). Comparative analysis of operating performance in "back Agriculture" and "non-back farm" types of agricultural listed companies. Journal of Anhui Agricultural Sciences, 35(33).

HU Li-xia. Some properties of lower-tail dependence copula. (2008). Journal of Lanzhou University (Natural Sciences), 44(5):108-111.

HUANG Yao, CAO Dong-xin. (2004). Research on equity financing of agricultural listed company. China 
Economist, (10):122-12.

Juri A,Wutrich M V. (2002). Copula convergence theorems for tail events. Insurance Mathematics and Economics, 30(3):405 420.

LI Yue, CHENG Xi-jun. (2006). Tail dependence analysis of SZI \& HSI based on Copula method. Systems Engineering, 24(5):88-92.

LIN Le-fen. (2004). An empirical study on agriculture listed companies in China. China Rural Survey, (6):66-70.

LIU Wei, YANG Yin-sheng. Evaluation and analysis of China's agricultural performance of listed companies. (2006). Journal of Agrotechnical Economics, (4):47-52.

LIU Zhu-lin, HU Yun-xia. (2008). Game analysis of the phenomenon of agricultural listed companies departing from agriculture. Journal of Anhui University of Technology (Social Sciences), 25(4):19-21.

REN Xian-ling, ZHANG Shi-ying. (2008). Tail dependence analysis of financial market based on the Copula. Statistics \& Information Forum, 23(6):6.

Sklar A. (1959). Fanction derepartitional dimensioned leursmarges. Publ.Inst.Stat.Univ.Paris, (8):229-231.

SUN Bei-bei, ZHONG Jian-xin. (2007). Countermeasures on improving the ability of raising money of agricultural public companies of China. Journal of Anhui Agricultural Sciences, 35(35): 11629-11631.

WANG Lu, WANG Qin, PANG Hao. (2007). Measuring and simulating the tail dependence of returns by Copula. Mathematics in Practice and Theory, 37(10):57-61.

WANG Xiao-fang,XIE Jin-jing. (2009) Comparison on tail dependence between SCI and SSTI based on Copulas.. Statistics \& Information Forum, 24(6):27-31.

ZHONG Jun, SHI Dao-ji. (2008). Tail dependence function of Shanghai and Shenzhen stock market returns. Mathematics in Practice and Theory, 38(10):5-12.

Table 1. Number of Four Categories of Listed Companies

\begin{tabular}{ccccc}
\hline Listing Location & Farming(number) & Forestry(number) & Fishery(number) & $\begin{array}{c}\text { Animal } \\
\text { husbandry(number) }\end{array}$ \\
\hline Shanghai & 9 & 2 & 2 & 1 \\
Shenzhen & 5 & 1 & 3 & 2 \\
total & 14 & 3 & 5 & 3 \\
\hline
\end{tabular}

Data of table 1 is from http://www.google.cn/finance/

Table 2. Parameter Estimation of Gumbel-H copula and K-S test

\begin{tabular}{cccc}
\hline Type & Valuation of Kendall $\tau$ & $\begin{array}{c}\text { non-parametric estimates } \\
\text { of } \theta\end{array}$ & K-S test \\
\hline SH-SZ & 0.2779 & 1.3849 & $1.0034(0.2664)$ \\
SHNY-SZNY & 0.4458 & 1.8045 & $0.8933(0.4021)$ \\
SHLY-SZLY & 0.1811 & 1.2212 & $0.8557(0.4567)$ \\
SHMY-SZMY & 0.1177 & 1.1334 & $0.6213(0.8348)$ \\
SHYY-SZYY & 0.1480 & 1.1736 & $0.6446(0.8004)$ \\
\hline
\end{tabular}

Note: SH-SZ denotes A-share agricultural listed companies in Shanghai and Shenzhen; SHNY-SZNY shows farming listed companies in Shanghai and Shenzhen; SHLY-SZLY says forestry listed companies in Shanghai and Shenzhen; SHYY-SZYY marks fishery listed companies in Shanghai and Shenzhen; SHMY-SZM shows animal husbandry listed companies in Shanghai and Shenzhen. The left value in K-S test a column K-S value, and the corresponding probability value is parentheses inside. 
Table 3. Parameter Estimation of Clayton copula and K-S test

\begin{tabular}{cccc}
\hline Type & Valuation of Kendall $\tau$ & $\begin{array}{c}\text { non-parametric estimates } \\
\text { of } \theta\end{array}$ & K-S test \\
\hline SH-SZ & 0.2779 & 0.7699 & $0.5719(0.8992)$ \\
SHNY-SZNY & 0.4458 & 1.6091 & $0.8009(0.5426)$ \\
SHLY-SZLY & 0.1811 & 0.4423 & $1.3643(0.0483)$ \\
SHMY-SZMY & 0.1177 & 0.2669 & $0.9910(0.2798)$ \\
SHYY-SZYY & 0.1480 & 0.3473 & $0.5890(0.8785)$ \\
\hline
\end{tabular}

Table 4. Calculators of upper and lower tail correlation coefficient

\begin{tabular}{|c|c|c|c|c|}
\hline \multirow{2}{*}{ Type } & \multicolumn{2}{|c|}{ Gumbel-H copula } & \multicolumn{2}{|c|}{ Clayton copula } \\
\hline & $\alpha$ & $\lambda_{U}$ & $\alpha$ & $\lambda_{L}$ \\
\hline \multirow{4}{*}{ SH-SZ } & 0.9500 & 0.3774 & 0.0500 & 0.4343 \\
\hline & 0.9750 & 0.3639 & 0.0250 & 0.4224 \\
\hline & 0.9950 & 0.3531 & 0.0050 & 0.4110 \\
\hline & $\alpha \rightarrow 1$ (left limit) & 0.3504 & $\alpha \rightarrow 0$ (right limit) & 0.4064 \\
\hline \multirow{4}{*}{ SHNY-SZNY } & 0.9500 & 0.5490 & 0.0500 & 0.6516 \\
\hline & 0.9750 & 0.5403 & 0.0250 & 0.6505 \\
\hline & 0.9950 & 0.5334 & 0.0050 & 0.6501 \\
\hline & $\alpha \rightarrow 1$ (left limit) & 0.5317 & $\alpha \rightarrow 0$ (right limit) & 0.6500 \\
\hline \multirow{4}{*}{ SHLY-SZLY } & 0.9500 & 0.2698 & 0.0500 & 0.2880 \\
\hline & 0.9750 & 0.2529 & 0.0250 & 0.2633 \\
\hline & 0.9950 & 0.2394 & 0.0050 & 0.2332 \\
\hline & $\alpha \rightarrow 1$ (left limit) & 0.2360 & $\alpha \rightarrow 0$ (right limit) & 0.2086 \\
\hline \multirow{4}{*}{ SHMY-SZMY } & 0.9500 & 0.1957 & 0.0500 & 0.1934 \\
\hline & 0.9750 & 0.1761 & 0.0250 & 0.1617 \\
\hline & 0.9950 & 0.1606 & 0.0050 & 0.1211 \\
\hline & $\alpha \rightarrow 1$ (left limit) & 0.1567 & $\alpha \rightarrow 0$ (right limit) & 0.0745 \\
\hline \multirow{4}{*}{ SHYY-SZYY } & 0.9500 & 0.2314 & 0.0500 & 0.2378 \\
\hline & 0.9750 & 0.2131 & 0.0250 & 0.2090 \\
\hline & 0.9950 & 0.1985 & 0.0050 & 0.1725 \\
\hline & $\alpha \rightarrow 1$ (left limit) & 0.1949 & $\alpha \rightarrow 0$ (right limit) & 0.1359 \\
\hline
\end{tabular}

